\title{
Subcutaneous Esketamine in a Case of Complex Post- Traumatic Stress Disorder: Case Report, a Negative Result
}

\author{
Carlos Gustavo Costardi, Mary SL Yeh, Felipe Branco Arcadepani, Matheus Souza Steglich, Acioly LT \\ Lacerda, Luciana Maria Sarin and Andrea Feijo Mello* \\ Department of Psychiatry, Federal University of São Paulo, São Paulo-SP, Brazil \\ *Corresponding author: Andrea Feijo Mello, Department of Psychiatry, CAISM Vila Mariana - Academic Building, Major \\ Maragliano, 241. Zip Code 04017-030, Sao Paulo-SP, Brazil
}

\begin{tabular}{|c|c|}
\hline ARTICLE INFO & ABSTRACT \\
\hline $\begin{array}{l}\text { Received: 蔧 July 26, } 2019 \\
\text { Published: 豐 August 12, } 2019\end{array}$ & $\begin{array}{l}\text { Keywords: Post-Traumatic Stress Disorder; Complex Post-Traumatic Stress Disorder; } \\
\text { Esketamine }\end{array}$ \\
\hline $\begin{array}{l}\text { tation: Carlos Gustavo Costardi, } \\
\text { ary SL Yeh, Felipe Branco Arcade- } \\
\text { Ini, Matheus Souza Steglich, Acioly LT } \\
\text { cerda, Luciana Maria Sarin, Andrea }\end{array}$ & $\begin{array}{l}\text { Abbreviations: PTSD: Post-Traumatic Stress Disorder; C-PTSD: Complex Post-Traumatic } \\
\text { Stress Disorder; MINI: Mini International Neuropsychiatric Interview Plus; MADRS: } \\
\text { Montgomery-Asberg Depression Rating Scale; YMRS: Young Mania Rating Scale; BSI: Beck } \\
\text { Scale for Suicide Ideation; CAPS-5: Clinician-Administered PTSD Scale for DSM-5; ICD-11: } \\
\text { International Classification of Diseases } 11^{\text {th }} \text { Review. }\end{array}$ \\
\hline
\end{tabular}
Feijo Mello. Subcutaneous Esketamine in a Case of Complex Post-Traumatic Stress Disorder: Case Report, a Negative Result. Biomed J Sci \& Tech Res 20(3)2019. BJSTR. MS.ID.003455.

\section{Case Report}

A 41-year-old woman was referred for psychiatric treatment at the Program for Research and Care on Violence and PTSD of The Federal University of São Paulo by her work supervisor. She worked as a nursing technician in an intensive care unit and presented with panic attacks at work after acknowledging about a physician's suicide with whom she had worked with. Eight months before the appointment to our service, the patient informed that she had witnessed the scene of her nephew's suicide at his house. She described this situation with intense distress, demonstrating sadness and guilt for not being able to help him. At her initial evaluation, she reported intrusive thoughts and flashbacks of her nephew's body, poor concentration and insomnia. She complained of nightmares, hypervigilance and irritability, avoidance of distressing memories and family activities. Patient was diagnosed with post-traumatic stress disorder (PTSD). The treatment consisted of fluoxetine $20 \mathrm{mg}$ per day, gradually increased to $80 \mathrm{mg} /$ day, and prazosin $2 \mathrm{mg} /$ day, which was not tolerated. In addition, she was referred to psychotherapy and withdrawal from work activities, however, she refused psychotherapy.
Patient reported that a month after the traumatic event, she sought treatment at a private setting. She was diagnosed with major depression and duloxetine $60 \mathrm{mg}$ daily was prescribed, but she quit treatment after four weeks due to lack of symptoms improvement. She had a prior history of sexual violence at age 25. At that time, she was treated for a short period and remained with significant social impairment for a long period of time. She denied alcohol or substance abuse. She denied symptoms of mania or psychosis. She had no family psychiatric history except for her nephew's suicide. She had no history of medical illness, or surgery. She lived in a house with her husband and her three sons. After three months of the present treatment, there was no evidence of any improvement. Several attempts to associate other medications such as risperidone, quetiapine, chlorpromazine and trazodone were tried. The patient's presentation became worse as symptoms of suicidal thoughts, interpersonal problems and emotional dysregulation, especially precipitated by family conflicts became more evident. She was also having dissociative symptoms, 
including depersonalization. In the following evaluations, she revealed that she had experienced childhood sexual abuse from age six to ten. She described a persistent sense of shame and guilt and reported that the current event brought many negative memories related to that trauma.

With this information, along with disturbances in self-concept and emotional dysregulation, diagnose was reviewed to complex post-traumatic stress disorder (C-PTSD) and comorbid major depressive disorder and current suicide risk, diagnosed by the Mini International Neuropsychiatric Interview Plus (MINI). It was then proposed to initiate weekly infusion of subcutaneous esketamine bolus injection into a study protocol which is approved by the Ethics Committee of The Federal University of São Paulo for treatment-resistant patients, targeting depressive and suicidal symptoms, which was accepted by the patient with consent of her family (Figures 1 \& 2).

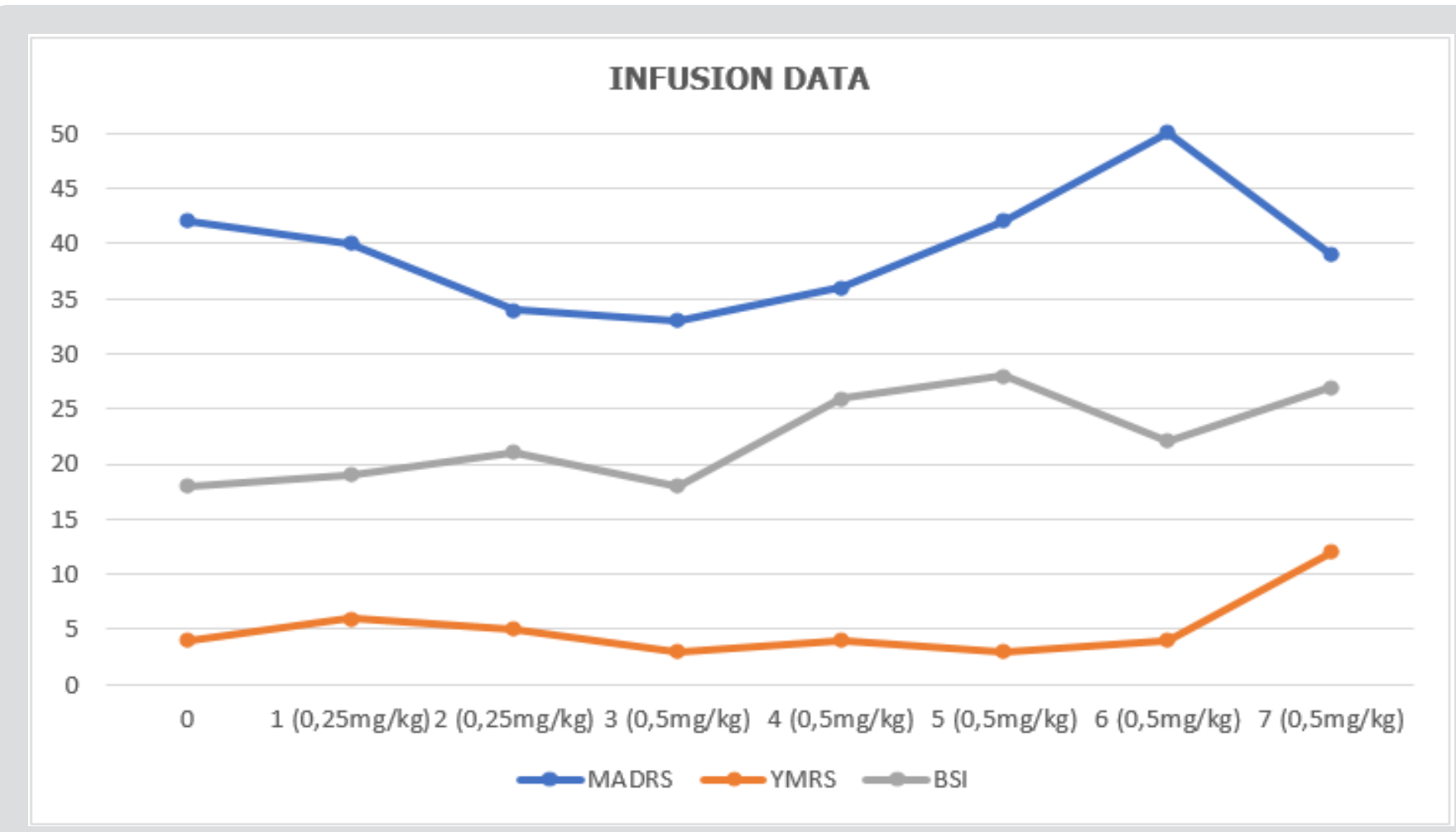

Figure 1: Shows infusion data, including dosage, Montgomery-Asberg Depression Rating Scale (MADRS), Young Mania Rating Scale (YMRS) and Beck Scale for Suicide Ideation (BSI).

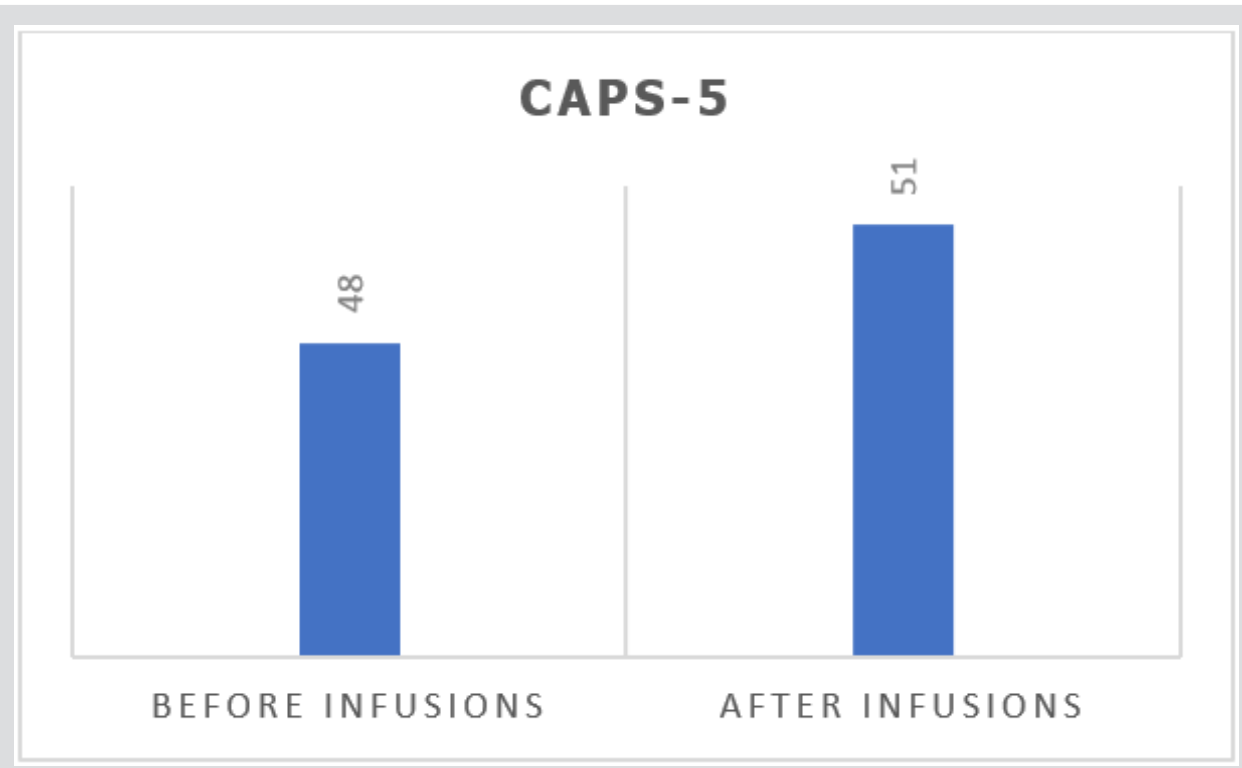

Figure 2: Shows Clinician-Administered PTSD Scale for DSM-5 (CAPS-5) before and after infusions. 
During infusions, the patient presented significant dissociation between thirty and forty-five minutes after onset, with psychomotor agitation, which was properly managed with verbal and space contention. It was observed that part of the experience during the dissociative episodes was related to the traumatic events of childhood. Continuity of treatment was then discussed with the patient. There was, however, difficulty in progressing ketamine dosage, which remained at $0,5 \mathrm{mg} / \mathrm{kg}$ due to the severity of dissociative symptoms. Despite showing an initial response, the patient remained severely symptomatic until the seventh infusion and it was decided to discontinue the esketamine treatment protocol since patient did not have improvement of depressive symptoms, nor PTSD.

\section{Discussion}

According to ICD-11 [1], complex PTSD is a disorder that may develop following exposure to an event of an extremely threatening, most commonly prolonged or repetitive events from which escape is difficult, including repeated childhood sexual abuse, as in the case presented here. It is considered a broader diagnosis that includes PTSD core symptoms plus an additional set of "disturbances in selforganization" symptoms, which are distributed in three clusters: affective dysregulation, negative self-concept, and disturbances in relationships $[2,3]$. There is extensive discussion in the literature regarding clinical aspects of C-PTSD and its common features with other diagnostic categories related to interpersonal trauma such as borderline personality disorder [3,4]. In addition, the high prevalence of comorbidity with other mental disorders imposes a challenge in the differential diagnosis [5]. In this case, the patient's symptoms of negative self-concept, emotional dysregulation and conflicting interpersonal relationships were more evident after longitudinal evaluation and observation of aspects of the clinical

ISSN: 2574-1241

DOI: 10.26717/BJSTR.2019.20.003455

Andrea Feijo Mello. Biomed J Sci \& Tech Res

This work is licensed under Creative Commons Attribution 4.0 License

Submission Link: https://biomedres.us/submit-manuscript.php history that had not been reported initially and raised a review on the patient's diagnosis and therapeutic strategies.

\section{Conclusion}

We consider relevant to present the case of a patient with complex PTSD compatible with the new diagnosis proposed by ICD-11, although esketamine infusions were not beneficial. Maybe the same pattern of limited response to treatment observed in patients with depressive symptoms with a history of severe early life trauma [6], with better response to psychotherapy associated treatment could be the best option, elaborating traumatic memories seems necessary. A prospective clinical trial comparing treatments with a significant number of subjects would be a suitable way of evaluating such cases.

\section{References}

1. (2018) World Health Organization (2018) International statistical classification of diseases and related health problems (11 ${ }^{\text {th }}$ Revision).

2. Hyland P, Shevlin M, Fyvie C, Karatzias T (2018) Posttraumatic Stress Disorder and Complex Posttraumatic Stress Disorder in DSM-5 and ICD11: Clinical and Behavioral Correlates. J Trauma Stress 31(2): 174-180.

3. Giourou E, Skokou M, Andrew SP, Alexopoulou K, Gourzis P, et al. (2018) Complex posttraumatic stress disorder: The need to consolidate a distinct clinical syndrome or to reevaluate features of psychiatric disorders following interpersonal trauma? World J Psychiatr 8(1): 12 19.

4. Cloitre M, Garvert DW, Weiss B, Carlson EB, Bryant RA (2014) Distinguishing PTSD, complex PTSD, and borderline personality disorder: A latent class analysis. Eur J Psychotraumatol 5: 10.

5. Karatzias T, Hyland P, Bradley A, Cloitre M, Roberts NP, et al. (2019) Risk factors and comorbidity of ICD-11 PTSD and complex PTSD: Findings from a trauma-exposed population based sample of adults in the United Kingdom. Depress Anxiety.

6. Nemeroff CB, Heim CM, Thase ME, Klein DN, Rush AJ, et al. (2003) Differential responses to psychotherapy versus pharmacotherapy in patients with chronic forms of major depression and childhood trauma. Proc Natl Acad Sci USA 100(24): 14293-14296.

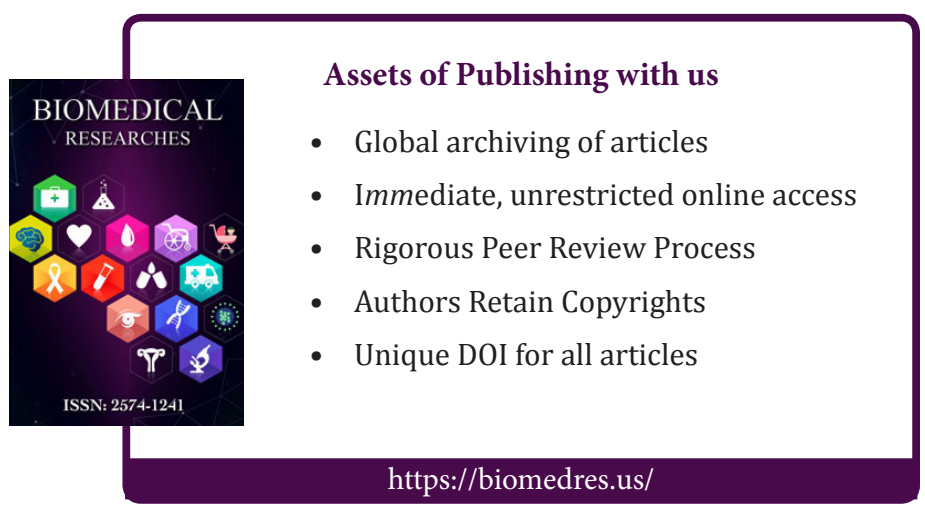

